\title{
The Red Queen's Race: An Experimental Card Game to Teach Coevolution
}

\author{
Amanda K Gibson ${ }^{1 *}$, Devin M Drown ${ }^{2}$ and Curtis M Lively ${ }^{1}$
}

\begin{abstract}
Although we are increasingly aware that an understanding of evolution is critical to all biological fields and to scientific literacy, evolution remains a challenge in the classroom. Here we present a hands-on, inquiry-based classroom activity to study host-parasite coevolution. Coevolution is the reciprocal evolution of interacting species. It is pervasive, diverse, and rapid. Instruction in coevolution is therefore an excellent way to teach students evolutionary principles. In the described game, students take on the role of either host or parasite, and they use playing cards to act out reciprocal selection. Students collaborate to collect data on the change in frequency of host and parasite genotypes (card suits) through time. They use these data to conduct an independent test of the prediction that hostparasite coevolution maintains genetic variation. The game is suitable for students ranging from upper-level high school through college. We include detailed instructions, discussion topics, and simple modifications to extend the game to additional topics. This is a fun, active, and simple exercise to introduce students to the complex topic of hostparasite coevolution. Moreover, the game emphasizes infectious diseases as major selective forces, a fascinating topic for today's students.
\end{abstract}

Keywords: Host-parasite coevolution, Rare advantage, Negative frequency-dependent selection, Red Queen Hypothesis, Sexual vs. asexual reproduction, Teaching evolution, Active learning

\section{Background}

Since its origins in the latter half of the nineteenth century, the field of evolutionary biology has made enormous strides. We now recognize evolution as a unifying framework for the biological sciences. There are more and more calls for the incorporation of evolutionary principles into applied fields (Denison et al. 2003; Antonovics et al. 2007; Read and Huijben 2009). For example, the evolutionary medicine movement seeks to incorporate evolutionary thinking into medical and pre-medical training (Nesse et al. 2010; Antolin et al. 2012). Education in evolutionary biology must grow to match our increasing awareness of the centrality of this field. Currently, the conceptual nature of evolution is notoriously difficult to teach, and the topic is fraught with misconceptions and complexity (Bishop and Anderson 1990; Alters and

\footnotetext{
*Correspondence: amakgibs@indiana.edu

${ }^{1}$ Department of Biology, Indiana University, Bloomington, IN 47405, USA Full list of author information is available at the end of the article
}

Nelson 2002; Dagher and Boujaoude 2005; Hokayem and BouJaoude 2008; Cunningham and Wescott 2009).

Thanukos (2010) recently made a compelling case that instruction on the topic of coevolution could tackle many of the conceptual hurdles that students face in understanding evolution. Coevolution is the reciprocal evolution of interacting species. It is pervasive, diverse, and very rapid. Instruction in coevolution can thus work to dispel the misconception that evolution acts only over long time-scales in response to abiotic changes, such as glaciation. Cases of coevolution demonstrate that evolution can occur over the course of only a few generations in response to continual change in the biotic environment. Moreover, coevolution powerfully contradicts the common notion of evolution as progressing towards a higher goal. In interactions between species, evolutionary optima are forever shifting in response to changes in the biotic environment (Jaenike 1978; Thompson 2005; Thanukos 2010).

In spite of this potential utility as a teaching tool in classrooms, Thompson (2010) notes that coverage of 
coevolution in biology textbooks is lacking. It is a challenging topic to teach via active laboratory exercises due to the complexity of balancing two or more interacting, adapting players. Here, we introduce a hands-on, inquiry-based activity to help students understand hostparasite coevolution. We developed it in order to provide students with a test of the Red Queen Hypothesis for the maintenance of genetic variation, which we discuss in more detail in the next section. Students work in small groups using playing cards to generate the coevolutionary trajectories of hosts and parasites. They personally discover, through the fate of their own card hands, the rapid rate of reciprocal adaptation and the potential for host-parasite interactions to maintain diversity.

\section{Host-Parasite Coevolution}

The Red Queen Hypothesis (RQH) predicts that coevolution between hosts and parasites acts to maintain genetic variation through time. We developed this activity so that students could test this prediction and, in doing so, work through a classic model of host-parasite coevolution. The $\mathrm{RQH}$ posits that parasites adapt to specifically infect the most common host genotypes in a population. Parasites thereby exert negative frequency-dependent selection on their hosts, with the most common host genotypes having low fitness and declining in frequency as parasites infect them. Rare host genotypes escape infection and increase in frequency (Haldane 1949; Jaenike 1978; Hamilton 1980; Hamilton et al. 1990). This rare advantage drives continual oscillations through time in the frequency of host genotypes and their matching parasite genotypes (Figure 1a) (Hutson and Law 1981; Nee 1989). Thus coevolution is proposed to maintain genetic diversity in host and parasite populations (Jaenike 1978; Bell 1982).

A key assumption of the $\mathrm{RQH}$ is that genetic matching is required for successful infection (Jaenike 1978). The most common model of infection genetics for studying Red Queen dynamics is the simple matchingalleles model (e.g. Hamilton et al. 1990; Howard and Lively 1994). This model evokes the idea of self-nonself recognition: infection occurs when the host fails to recognize the parasite as a foreign entity. A host is susceptible to infection (i.e. the parasite is successful) if the host and parasite have matching genotypes. In contrast, a host is resistant to infection (i.e. the parasite is unsuccessful) if host and parasite genotypes are mismatched (Figure 1b). Under this model, there is a strong trade-off to specialization, such that a parasite genotype is infective to only a fraction of host genotypes (Frank 1993).

The RQH itself is a powerful educational tool. First, host-parasite coevolution demonstrates to students that

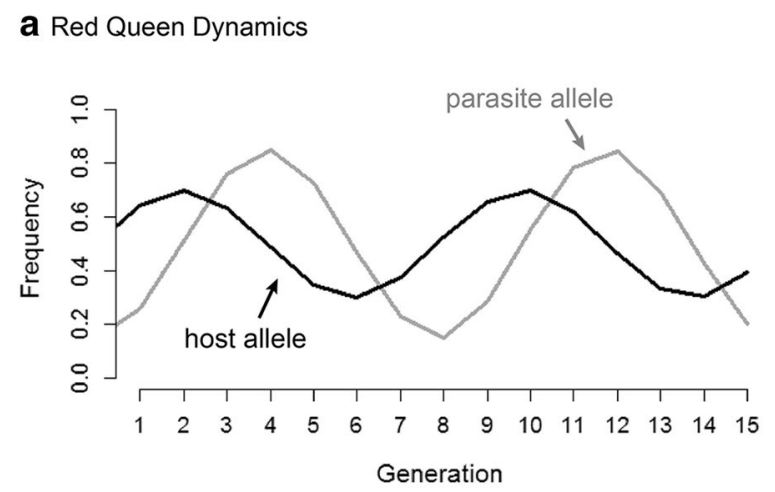

b Matching-Alleles Model

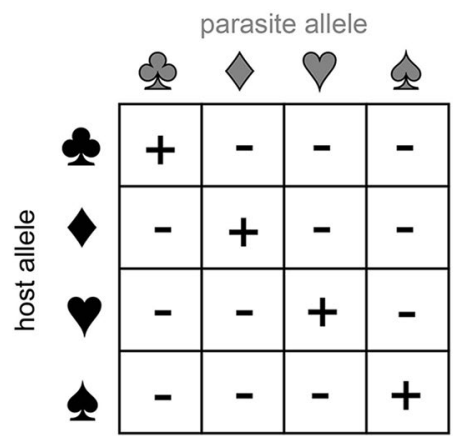

Figure 1 Red Queen model of host-parasite coevolution. a Schematic of oscillations through time in the frequency of a host genotype (black) and its matching parasite genotype (gray). Host genotypes decline when common and increase when rare due to negative frequency-dependent selection exerted by the coevolving parasite population. The time-lag in the parasite genotype frequency reflects the period required for the parasite population to adapt to the changing host population. b Diagram of the matching-alleles model, as implemented in the game. Successful infection (+) results when the parasite genotype, represented here by a single allele or card suit, matches the host genotype. The host resists infection (-) when the host and parasite allele are mismatched.

evolution can be a rapid, dynamic process. Secondly, the $\mathrm{RQH}$ focuses on parasites and infectious diseases, a timely and engaging topic. The topic particularly appeals to biomedical students, who make up a large fraction of biology majors. An understanding of infectious disease biology is of grave importance in today's world. The public are much more aware of disease outbreaks in human and non-human populations. The RQH can help students understand these events: it teaches the basics of infection genetics and makes predictions for the evolution of host and parasite populations over the course of epidemics. Similarly, the rise of antibiotic resistance is both a striking example of modern-day evolution in action and a pressing public health threat (Task Force for Combating Antibiotic-Resistance Bacteria 2015). The RQH can teach students some key evolutionary aspects of the problem: populations respond rapidly to strong selection, 
and parasites relentlessly adapt to host defenses. It is our moral obligation to train students on these issues, particularly students destined for medical professions (Antonovics et al. 2007; Read and Huijben 2009; Nesse et al. 2010; Antolin et al. 2012). We feel that teaching host-parasite coevolution in the context of the RQH is an excellent way to accomplish this goal. We propose a classroom activity that is inexpensive, fun, and enlightening. The game provides an entry point for students to discover the basics of host-parasite coevolution.

\section{The Red Queen's Game}

Our game is effectively a simulation in which students take on the roles of host and parasite populations. This role-playing is the key to student engagement and comprehension. Students playing the host not only see the oscillations in genotype frequency in the data they generate: they personally dread the adaptation of the parasite population to their host hand and suffer the inevitable crash of the host population. Likewise, students playing the parasite take satisfaction from the growing success of the parasite population as it adapts to the host population and lament its failure as the composition of the host hand rapidly shifts away in response. We present the game to the students as a means to test the key prediction of the $\mathrm{RQH}$ : host-parasite coevolution maintains genetic variation. The students use the game to see for themselves if this prediction is upheld. In other words, the students are engaged in hypothesis testing.

Our goal for this exercise is to convey four general concepts (Figure 2a): (1) coevolution occurs rapidly; (2) that which is most fit now can become the least fit in just a few generations; (3) rare advantage, or negative frequency-dependent selection, can maintain genetic variation over time; and (4) we can use simple games to represent complex processes and to test hypotheses. Prior to beginning the game, we encourage instructors to pose four questions to their students that will emphasize these concepts. Presented in Figure 2b, these "warm-up" questions ask students to reflect upon their initial understanding of coevolution and how we might study it. They will struggle to answer these questions prior to playing the game. By guessing and discussing answers with their peers, however, students will be thinking about the key concepts of the game as they begin playing.

\section{Materials}

The only required materials for the game are two decks of playing cards per group of students, one deck for the host population and one for the parasite population. It is best to use two distinct decks to facilitate separation of the host and parasite populations (e.g. blue-backed decks for host, red-backed for parasite). We have made additional resources available online at http://www.indiana.edu/ curtweb/EvolutionLabs/ (Lively 2015) and in Additional files 1 and 2 (a worksheet with directions and a spreadsheet for data entry and calculations). We provide the spreadsheet to students as a Google sheet and give all students access and editing privileges. Students enter their data directly into the Google sheet, and we project the results of the game live. This set-up facilitates sharing of the data between all groups. It requires that each group of students have access to a computer for data entry.

\section{Game Set-Up}

We present brief introductory material to the students and then instruct them to split into groups, collect card decks, and read the directions thoroughly. We conduct this exercise with students in groups of two: one student playing the host population and one playing the parasite population. Students are advised to switch halfway through so that they can personally experience both roles. We suggest students play for 15 generations, which requires a minimum of $45 \mathrm{~min}$.

The sequence of the game is simple, and students catch on after 1-2 generations of independent play. The game is amenable to modification according to the level and size of the class and to the desired learning goals. Here, we outline the specific approach that we have used for playing the game with 24 undergraduate students in CM Lively's Evolution course at Indiana University.

Students begin by establishing their starting host and parasite populations. They shuffle their respective decks and randomly select 12 cards. The remaining cards become the reserve deck. Each suit is a genotype-clubs, spades, hearts, and diamonds. Students count the number of individuals of each "genotype" in their hand and record these data in the generation 0 row of their data sheet (spreadsheet provided online and in Additional file 2). Students should ensure that these counts sum to 12 for both the host and parasite populations: the provided spreadsheet includes a column for this purpose. We additionally constructed the spreadsheet such that counts are automatically translated into frequencies and plotted. For example, if half of the cards held by the "host" are spades, then the frequency of the spade genotype is 0.5 .

Four basic steps constitute a single "generation" of this game (Figure 3):

Step 1: host-parasite contact Host and parasite shuffle their populations. They then work together to randomly pair each host and parasite card, resulting in 12 host-parasite pairs. Students tend to find their own way of efficiently performing this step. For example, the host student could lay out her cards, and then the parasite 


\section{a Concepts}

1. Coevolution is rapid

2. That which is most fit now can soon become the least fit

3. Rare advantage maintains genetic variation over time

4. Simple games can capture complex processes and test hypotheses

\section{b Warm-up questions}

1. How fast is coevolution? How many generations (i.e. rounds of play) do you expect it to take to see evolutionary change in the host or parasite population: ten? One thousand? One million?

2. How would you find the most fit genotype in a population? How would you measure change in fitness over time?

3. Do you think natural selection favoring rare genotypes will maintain genetic variation in a population? Why? How would you test this?

4. What factors impact species interactions in nature? How?

\section{Wrap-up questions}

1. Have the results of the game changed your views on the rate of coevolution? Why?

- Compare the oscillations of your host and parasite genotype frequencies. For a given suit, are the oscillations of host and parasite perfectly in sync? If not, is host or parasite lagging, and by how many generations? Why do you think this might be?

2. Now, with data in hand, how would you propose we assess whether a genotype is the most fit in a population? And how would you measure changes in fitness over time?

- Calculate the change in frequency of each host genotype from generations 1 to 4 . Which genotype increases the most?

- Calculate the change in frequency of each host genotype from generations 4 to 8 . Which genotype increases the most? Is it the same genotype as the previous answer? Have any genotypes switched from increasing to decreasing, or vice versa?

3. Did natural selection favor rare genotypes in this game? Why or why not? How would you propose we assess whether or not genetic variation was maintained?

- Apply your suggestion - was genetic variation maintained? Consider genetic variation in both the host and the parasite population in your answer.

- What do you predict would happen to host genotype frequencies over time if parasites were absent? Sketch your prediction. Design a modification of the game to test your prediction.

4. Do you think all coevolution can be described by these "Red Queen dynamics?" What's an alternative? You previously listed factors that might impact species interactions in nature - if you incorporated them into the game, how might your results differ?

\section{d Metapopulation thinking}

- Why do the individuals populations differ so dramatically from one another? Why does the metapopulation differ so dramatically from that of the individual populations?

- Based upon the metapopulation data, is any one genotype inherently more fit than another?

- Imagine that we allowed occasional migration between populations: on average, would a migrant host's fitness be greater or less than the local hosts? A migrant parasite's?

Figure 2 Concepts and questions for classroom discussion. We outline a four central concepts of the game, $\mathbf{b}$ warm-up questions to emphasize these concepts, c wrap-up questions in which students revisit and revise their responses to the warm-up questions, and $\mathbf{d}$ questions for discussing the metapopulation level of the game.

student can lay out his cards next to the host cards until 12 pairs are formed. This step requires that each group have enough work space to lay out their pairs. It is also helpful for host and parasite decks to be distinct in some way (e.g. different backs, say red and blue), so that host and parasite individuals can be separated following the selection step.

Step 2: infection and selection According to the matching-alleles model for infection genetics, a parasite successfully infects a host when its genotype matches that of its host (Frank 1993). Therefore, infection results for those pairs in which the host and parasite genotype match (e.g. host and parasite are both spades) (Figure 1b). The host individual is sterilized or killed, so the host student discards that card by placing it in her reserve deck. The parasite student retains the successful parasite card for subsequent reproduction. For pairs in which the host and parasite genotype do not match, the host resists infection. The parasite does not survive the failed infection (as in Salathé et al. 2008; King et al. 2011), so the parasite student discards it by placing it in his reserve deck. The host student retains the successful 


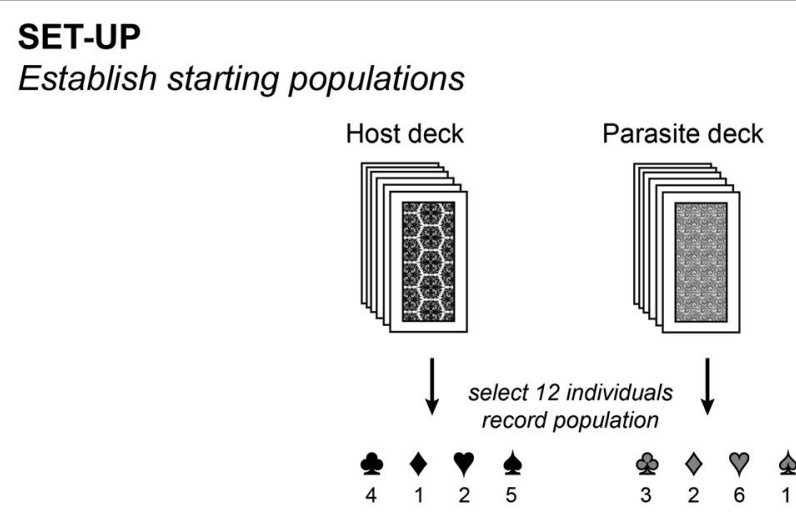

\section{STEP 1}

Host-parasite contact

Host
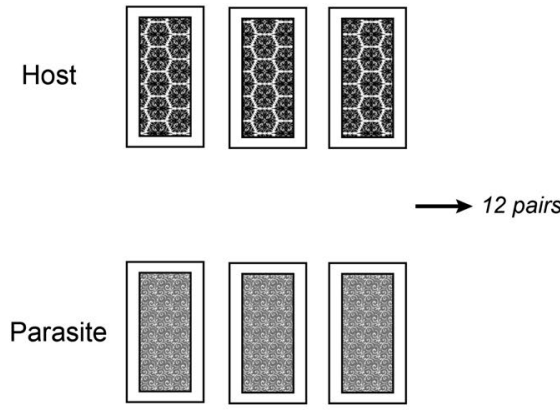

\section{STEP 3}

\section{Reproduction}
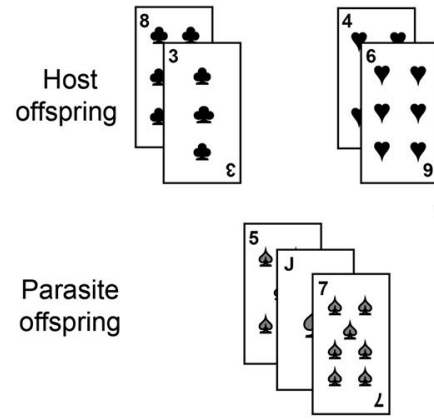

Too few matching cards in reserve? Mutation: randomly select additional offspring

\section{STEP 2}

Infection and selection

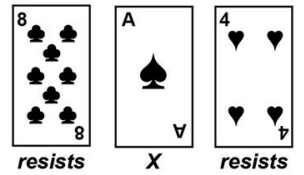

$\longrightarrow 12$ pairs
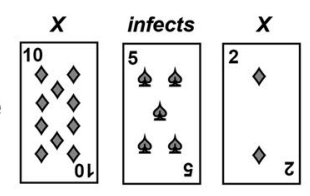

\section{STEP 4}

Population size regulation

Less than 12 offspring? More than 12 offspring?

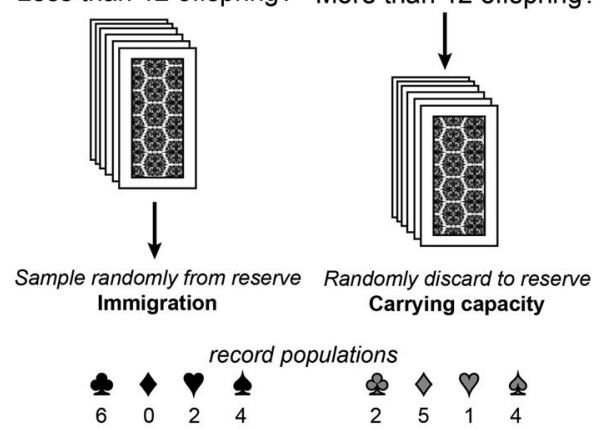

Figure 3 Schematic of the set-up and four basic steps of the Red Queen game. These four steps constitute a single generation of play. We propose 15 generations of play (i.e. 15 repetitions of these steps) for a classroom exercise.

host card for reproduction. In this formulation of the game, each parasite individual has only this single chance to infect. Students will often find that matches (successful infections) are rare in the initial generations of the game and increase through time as the parasite population adapts.

Step 3: reproduction Each surviving host makes two offspring and dies. Students simulate this process by adding one card of the matching suit for each surviving host card (for a total of two cards of the same suit).
Each successful parasite makes three offspring and dies. Students simulate this process by adding two cards of the matching suit for each surviving parasite card (for a total of three cards). Sometimes, a student's reserve deck does not have enough cards of a given suit to give each surviving individual enough offspring. In this case, the student should randomly select cards from the reserve deck until all individuals have reproduced. These randomly selected offspring will not match the genotype of the parent; students can think of this step as mutation. 
We feel that the greater offspring number of parasites relative to hosts ( 3 vs. 2 ) is biologically realistic. Computer simulations also demonstrated that this tends to generate smoother oscillatory dynamics than equivalent offspring numbers: it increases the probability of matching by facilitating rapid evolution of the parasite (data not shown).

Step 4: population size regulation Students rarely have exactly 12 individuals at the end of the reproduction step. The population nonetheless remains fixed at 12. If populations have too few offspring (common for the parasite population in particular), students should randomly select offspring from the reserve deck until they have 12 offspring. This step can be thought of as immigration. If populations have too many offspring (common for the host population), students should shuffle the offspring and randomly select 12 cards to make the next generation. They should return the remainder to their reserve deck. This step is consistent with a carrying capacity for the population. The students then record the number of individuals of each genotype under generation 1 of the spreadsheet. Repeat steps 1-4 for 14 more generations. We find that 15 generations is sufficient to obtain 3-4 oscillations (Figure 4).

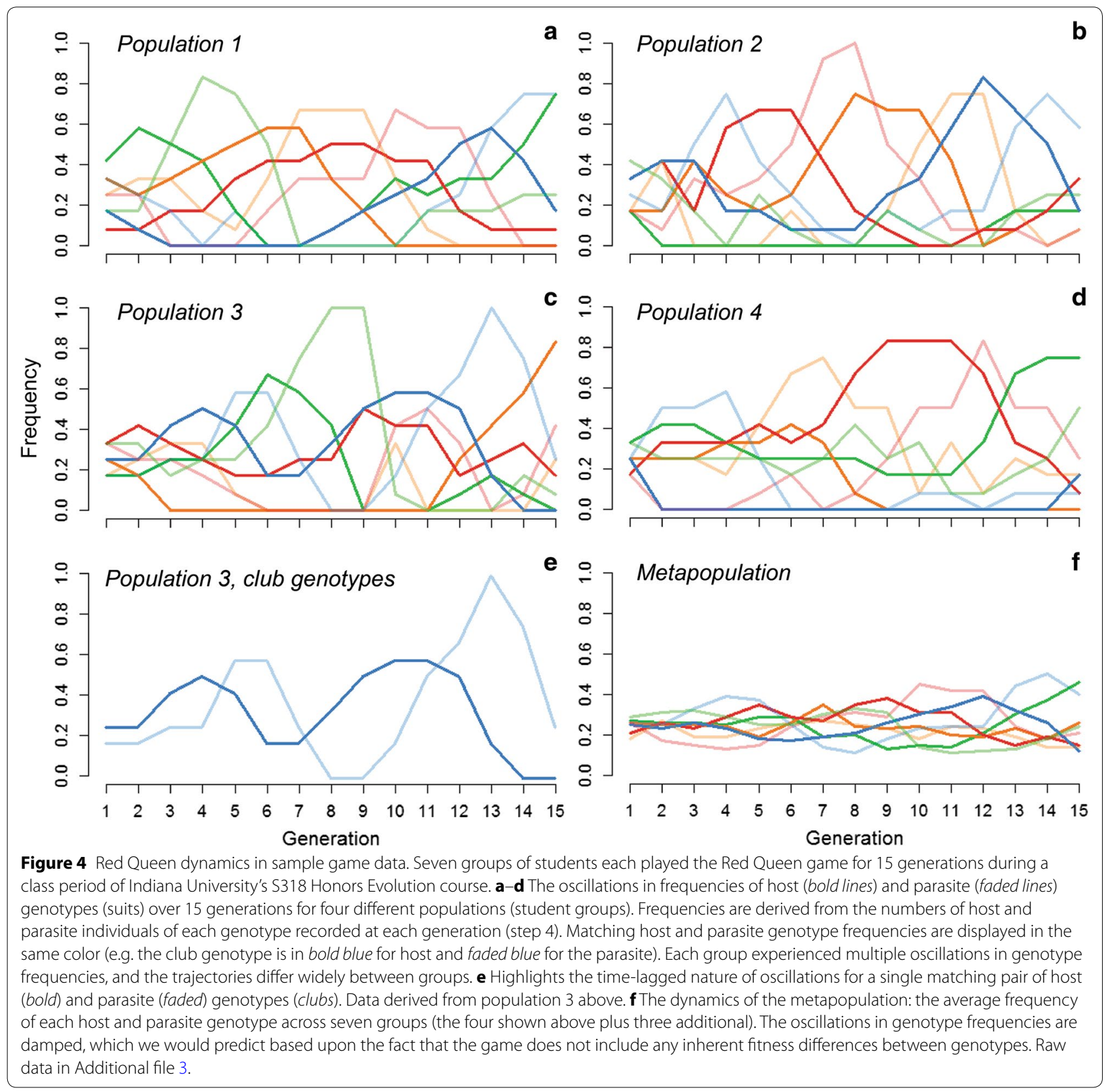




\section{Outcome}

After 15 generations, the students will have generated host and parasite genotype frequencies through time. If using a data entry system that allows live updates and data sharing, each group will also have access to the data and plots of other groups in the class. In Figure 4, we show sample data generated by our own students (raw data provided in Additional file 3). Oscillations in host and parasite genotype frequencies, with a timelag of a few generations, are obvious (Figure $4 a-d)$. The specific follow-up exercises that an instructor wishes to follow should be tailored to the level of the students, the prior coverage of these topics in the class, and the instructor's specific educational goals. We propose several questions to return students to the game's key concepts. Presented in Figure 2c, these "wrap-up" questions ask students to revisit, and perhaps revise, their answers to the warm-up questions (Figure 2b). In answering them, students use the data they've generated to measure time lags in parasite adaptation, changes in host fitness over time, and genetic variation in the host and parasite populations.

We also encourage instructors to show students the results at the level of the "metapopulation," meaning across all populations. We propose this for several reasons. First, coevolution leads to divergence between populations: as a host and parasite population reciprocally adapt, they can adopt distinct evolutionary trajectories from their neighbors, just by chance alone. Students will see this when they compare allele frequencies at generation 15 in different groups (Figure $4 a-d)$. Secondly, no genotype has an inherent fitness advantage in the game: fitness is determined solely by the frequency of a genotype's matching partner. This is an unusual idea that is obvious in the metapopulation data: each host and parasite genotype is maintained at $\sim 25 \%$ of the metapopulation, and the oscillations are damped (Figure 4f). Finally, we have come to realize that coevolution must be considered at the metapopulation level (Thompson 2005): for example, moderate gene flow between populations can promote coevolution by increasing genetic variation (Gandon et al. 1996; Lively 1999; Gandon and Michalakis 2002; Greischar and Koskella 2007). This exercise exposes students to this kind of metapopulation thinking. The provided spreadsheet for data entry (Additional file 2) includes a tab to calculate and plot the average host and parasite genotype frequencies across all groups of students (Figure 4f). In Figure 2d, we present three discussion questions that highlight these key metapopulation points: the striking divergence between populations, equal mean fitness of all genotypes, and migration between populations.

\section{Extensions}

\section{Discussion Topics}

We recommend several published articles for discussion that would pair nicely with the above activity. For example, Chaboudez and Burdon (1995), Lively et al. (1990), and Wolinska and Spaak (2009) provide simple and compelling studies of frequency-dependent selection by parasites in natural systems. Chaboudez and Burdon (1995) surveyed natural populations of a flowering plant infected with a rust fungus to test the Red Queen's prediction that parasites adapt to infect the most common host clones in a population. Their data support this prediction: the most common clone was the only infected clone in the majority of host populations, a greater fraction than expected by random chance. Students could apply the Chaboudez and Burdon (1995) approach to their simulated data: at generation 15 , in what proportion of populations is the most common host genotype matched by the most common parasite genotype?

We developed this activity in conjunction with a unit on the evolution of sexual reproduction. The RQH arose to address the paradox of sexual reproduction (Jaenike 1978; Bell 1982). Asexual reproduction is far more efficient than sexual reproduction, so asexual lineages should outcompete sexual ones (Maynard Smith 1978; Lively and Lloyd 1990; Lively 1996). Why then is sexual reproduction so common? The Red Queen argues that host-parasite coevolution favors sexual individuals because they produce offspring with a variety of rare genotypes. In contrast, parasites rapidly adapt to infect common asexual lineages and drive them down in frequency (Haldane 1949; Jaenike 1978; Hamilton 1980; Hamilton et al. 1990). To further address hypotheses for the maintenance of sex and genetic variation, we recommend Burt and Bell (1987). Their study provides an excellent example of the strong inference approach to hypothesis testing. It also encourages students to reflect upon the evolutionary conditions under which we predict selection for elevated recombination. Lastly, Hamilton and Zuk (1982) is a classic work that would serve to connect host-parasite coevolution to a unit on sexual selection.

\section{Variations on the Game}

An exciting aspect of the game we outline here is that the basic framework can be modified in small ways to test additional predictions. Instructors can ask students to design their own modifications to test new predictions they have made based upon class data (e.g. Figure 2c, question 3). Here, we highlight four of the many modifications that might be interesting to pursue in the classroom.

First, to drive home the idea that coevolution acts to maintain genotypic diversity, the students can contrast 
the original game with one in which selection is absent. In this case, students play the game as before, but there are no fitness consequences of successful or unsuccessful infection for either host or parasite. In other words, parasite virulence is zero, and there is no cost for a parasite of a failed infection. Students should predict in advance that genetic variation will not be maintained under these conditions, because rare advantage is absent.

Secondly, students can further test the advantage of a rare host genotype by beginning the game with no genetic variation. In this modification, the game starts with a single suit and variation is introduced through mutation (in step 3) and migration (in step 4). Students should predict in advance that mutant or migrant host genotypes will rapidly increase in frequency. In contrast, mutant or migrant parasite genotypes will be less successful, as they are unlikely to match the most common host genotype.

This suggests a third modification: for advanced classes, instructors might consider introducing the concept of local adaptation using data generated by the game. Coevolution is predicted to result in local adaptation, in which parasites have higher fitness when infecting their sympatric host population than allopatric host populations (Parker 1985; Lively 1989). By calculating mean fitness of each parasite population on the host population of each group at generation 15, we indeed find local adaptation of parasite populations (data not shown).

A final option is to explicitly test the Red Queen's prediction for the maintenance of sexual reproduction: coevolving parasites maintain sex in their hosts. In the game described here, all host individuals are clonal. Step 3 (reproduction) might be modified to compete sexual individuals against clones: a fraction of host individuals could be clonal, with the rest producing offspring in a manner that models recombination. There are of course many modifications besides these few that we highlight here: the game could be altered to use diploid rather than haploid individuals or to test the results obtained under different virulence levels. The option also exists to use custom card decks, rather than traditional playing cards, to have greater flexibility in the number of genotypes and individuals per genotype.

\section{Conclusion}

Pedagogical studies in biology and general science emphasize the efficacy of hands-on, inquiry-based activities that actively engage students in the learning process (Hake 1998; Alters and Nelson 2002; Smith et al. 2005; Nelson 2008). We have described a game in which students work cooperatively in small groups to generate their own data for an independent test of the central prediction of the Red Queen Hypothesis. In pursuing this specific goal, students personally engage with the broader concepts of rapid coevolution and frequency-dependent selection. We offer this basic exercise as a fun and inexpensive tool for teaching evolution at the undergraduate and advanced high school level.

\section{Additional files}

Additional file 1: Worksheet with directions and discussion questions.

Additional file 2: Spreadsheet for data entry and calculations.

Additional file 3: Sample classroom data presented in Figure 4

\section{Abbreviation}

$\mathrm{RQH}$ : Red Queen Hypothesis.

\section{Author's Contributions}

AKG designed the activity, facilitated data collection, and wrote the manuscript. DM Drown improved upon the activity and contributed to the manuscript. CM Lively designed the activity, facilitated data collection, and contributed to the manuscript. All authors read and approved the final manuscript.

\section{Author details}

${ }^{1}$ Department of Biology, Indiana University, Bloomington, IN 47405, USA. ${ }^{2}$ Institute of Arctic Biology, University of Alaska Fairbanks, Fairbanks, AK 99775 , USA.

\section{Acknowledgements}

We first and foremost thank the students of Indiana University's Honors Evolution S318 course in the fall of 2014 for inspiring this activity, test driving it, and giving helpful feedback. We are grateful to Amrita Bhattacharya for helping test early versions of the game, as well as Amy Dapper, Marta Shocket, and Mikus Abolins-Abols for their valuable input. AK Gibson recognizes funding from the National Science Foundation's Graduate Research Fellowship Program. We thank Albert and Kathy Ruesink for establishing the Albert Ruesink Outstanding Associate Instructor Teaching Award in Biology. This award to AKG contributed to publication costs.

\section{Compliance with Ethical Guidelines}

Competing interests

The authors declare that they have no competing interests.

Received: 14 May 2015 Accepted: 11 July 2015

Published online: 21 July 2015

\section{References}

Alters, B. J., \& Nelson, C. E. (2002). Perspective: teaching evolution in higher education. Evolution, 56, 1891-1901.

Antolin, M. F., Jenkins, K. P., Bergstrom, C. T., Crespi, B. J., De, S., Hancock, A., et al. (2012). Evolution and medicine in undergraduate education: a prescription for all biology students. Evolution, 66, 1991-2006.

Antonovics, J., Abbate, J. L., Baker, C. H., Daley, D., Hood, M. E., Jenkins, C. E., et al. (2007). Evolution by any other name: antibiotic resistance and avoidance of the e-word. PLoS Biology, 5, e30.

Bell, G. (1982). The masterpiece of nature: the evolution and genetics of sexuality. Berkeley: University of California Press.

Bishop, B. A., \& Anderson, C. W. (1990). Student conceptions of natural selection and its role in evolution. Journal of Research in Science Teaching, 27, $415-427$.

Burt, A., \& Bell, G. (1987). Mammalian chiasma frequencies as a test of two theories of recombination. Nature, 326, 803-805.

Chaboudez, P., \& Burdon, J. (1995). Frequency-dependent selection in a wild plant-pathogen system. Oecologia, 102, 490-493. 
Cunningham, D., \&Wescott, D. (2009). Still more "fancy" and "myth" than "fact" in students' conceptions of evolution. Evolution: Education and Outreach, 2, 505-517.

Dagher, Z. R., \& Boujaoude, S. (2005). Students' perceptions of the nature of evolutionary theory. Science Education, 89, 378-391.

Denison, R., Kiers, E., \& West, S. (2003). Darwinian agriculture: when can humans find solutions beyond the reach of natural selection? Quarterly Review of Biology, 78, 145-168.

Frank, S. A. (1993). Specificity versus detectable polymorphism in host-parasite genetics. Proceedings of the Royal Society B: Biological Sciences, 254, 191-197.

Gandon, S., Capowiez, Y., Dubois, Y., Michalakis, Y., \& Olivieri, I. (1996). Local adaptation and gene-for-gene coevolution in a metapopulation model. Proceedings of the Royal Society B: Biological Sciences, 263, 1003-1009.

Gandon, S., \& Michalakis, Y. (2002). Local adaptation, evolutionary potential and host-parasite coevolution: interactions between migration, mutation, population size and generation time. Journal of Evolutionary Biology, $15,451-462$.

Greischar, M. A., \& Koskella, B. (2007). A synthesis of experimental work on parasite local adaptation. Ecology Letters, 10, 418-434.

Hake, R. R. (1998). Interactive-engagement versus traditional methods: a sixthousand-student survey of mechanics test data for introductory physics courses. American Journal of Physics, 66, 64-74.

Haldane, J. B. S. (1949). Disease and evolution. Ricerca Scientifica, 19(Suppl. 1), 68-76.

Hamilton, W. D. (1980). Sex versus non-sex versus parasite. Oikos, 35, 282-290.

Hamilton, W. D., Axelrod, R., \&Tanese, R. (1990). Sexual reproduction as an adaptation to resist parasites (a review). Proceedings of the National Academy of Sciences, 87, 3566-3573.

Hamilton, W. D., \& Zuk, M. (1982). Heritable true fitness and bright birds: a role for parasites? Science, 218, 384-387.

Hokayem, H., \& BouJaoude, S. (2008). College students' perceptions of the theory of evolution. Journal of Research in Science Teaching, 45, 395-419.

Howard, R. S., \& Lively, C. M. (1994). Parasitism, mutation accumulation and the maintenance of sex. Nature, 367, 554-557.

Hutson, V., \& Law, R. (1981). Evolution of recombination in populations experiencing frequency-dependent selection with time delay. Proceedings of the Royal Society B: Biological Sciences, 213, 345-359.

Jaenike, J. (1978). An hypothesis to account for the maintenance of sex within populations. Evolutionary Theory, 3, 191-194.

King, K. C., Jokela, J., \& Lively, C. M. (2011). Trematode parasites infect or die in snail hosts. Biology Letters, 7, 265-268.

Lively, C. M. (1989). Adaptation by a parasitic trematode to local populations of its snail host. Evolution, 43, 1663-1671.

Lively, C. M. (1996). Host-parasite coevolution and sex. BioScience, 46, 107-114.
Lively, C. M. (1999). Migration, virulence, and the geographic mosaic of adaptation by parasites. The American Naturalist, 153, S34-S47.

Lively, C. M. (2015). Evolution Labs for Teachers. Indiana University. http://www. indiana.edu/ curtweb/EvolutionLabs/.

Lively, C. M., Craddock, C., \&Vrijenhoek, R. C. (1990). Red Queen hypothesis supported by parasitism in sexual and clonal fish. Nature, 344, 864-866.

Lively, C. M., \& Lloyd, D. G. (1990). The cost of biparental sex under individual selection. The American Naturalist, 135, 489-500.

Maynard Smith, J. (1978). The evolution of sex. Cambridge: Cambridge University Press.

Nee, S. (1989). Antagonistic coevolution and the evolution of genotypic randomization. Journal of Theoretical Biology, 140, 499-518.

Nelson, C. E. (2008). Teaching evolution (and all of biology) more effectively: strategies for engagement, critical reasoning, and confronting misconceptions. Integrative and Comparative Biology, 48, 213-225.

Nesse, R. M., Bergstrom, C. T., Ellison, P. T., Flier, J. S., Gluckman, P., Govindaraju, D. R., et al. (2010). Making evolutionary biology a basic science for medicine. Proceedings of the National Academy of Sciences, 107, 1800-1807.

Parker, M. A. (1985). Local population differentiation for compatibility in an annual legume and its host-specific fungal pathogen. Evolution, 39, $713-723$.

Read, A. F., \& Huijben, S. (2009). Perspective: Evolutionary biology and the avoidance of antimicrobial resistance. Evolutionary Applications, 2, 40-51.

Salathé, M., Kouyos, R. D., Regoes, R. R., \& Bonhoeffer, S. (2008). Rapid parasite adaptation drives selection for high recombination rates. Evolution, 62, 295-300.

Smith, K. A., Sheppard, S. D., Johnson, D. W., \& Johnson, R. T. (2005). Pedagogies of engagement: Classroom-based practices. Journal of Engineering Education, 94, 87-101.

Task Force for Combating Antibiotic-Resistance Bacteria. (2015). National Action Plan for Combating Antibiotic-Resistant Bacteria. The White House. https://www.whitehouse.gov/sites/default/files/docs/national_ action_plan_for_combating_antibotic-resistant bacteria.pdf.

Thanukos, A. (2010). Coevolution in the classroom. Evolution: Education and Outreach, 3, 71-77.

Thompson, J. N. (2005). The geographic mosaic of coevolution. Chicago: University of Chicago Press.

Thompson, J. N. (2010). Four central points about coevolution. Evolution: Education and Outreach, 3, 7-13.

Wolinska, J., \& Spaak, P. (2009). The cost of being common: evidence from natural Daphnia populations. Evolution, 63, 1893-1901.

\section{Submit your manuscript to a SpringerOpen ${ }^{\odot}$ journal and benefit from:}

- Convenient online submission

- Rigorous peer review

- Immediate publication on acceptance

- Open access: articles freely available online

- High visibility within the field

- Retaining the copyright to your article

Submit your next manuscript at springeropen.com 